\title{
Voronoi Diagram and Fast Marching applied to Path Planning
}

\author{
Santiago Garrido \\ Robotics Lab. \\ Carlos III University \\ Madrid, Spain \\ Email: sgarrido@ing.uc3m.es
}

\author{
Luis Moreno \\ Robotics Lab. \\ Carlos III University \\ Madrid, Spain \\ Email: moreno@ing.uc3m.es
}

\author{
Dolores Blanco \\ Robotics Lab. \\ Carlos III University \\ Madrid, Spain \\ Email: dblanco@ing.uc3m.es
}

\begin{abstract}
This paper presents a new Path Planning method which operates in two steps. In the first step the safest areas in the environment are extracted by means of a Voronoi diagram. In the second step Fast Marching Method is applied to the Voronoi extracted areas in order to obtain the shortest path. In this way the trajectory obtained is the shortest between the safe possible ones. This two step method combines speed and reliability, because the map dimensions is reduced to a unidimensional map and this map represents the safest areas in the environment for moving the robot.
\end{abstract}

\section{INTRODUCTION}

When we want to move a robot from a place to another place it is necessary a global map to calculate a global trajectory. Mobile robot path planning approaches can be divided into five classes [1]. Roadmap methods extract a network representation of the environment and then apply graph search algorithms to find a path. Exact cell decomposition methods construct non-overlapping regions that cover free space and encode cell connectivity in a graph. Approximate cell decomposition is similar, but cells are of predefined shape (e.g. rectangles) and do not exactly cover free space. Potential field methods differ from the other four in that they consider the robot as a point evolving under the influence of forces that attract it to the goal while pushing it from obstacles. Navigation functions are commonly considered a special case of potential fields.

In order to calculate the trajectory in the global map, this paper presents a new Path Planning method based in the combination of Voronoi Diagram and the Fast Marching Method.

The Fast Marching Method has been applied to Path Planning [Sethian:1], and their trajectories are of minimal distance, but they are not very safe because the path is too close to obstacles and what is more important, the path is not smooth enough.

In order to improve the safety of the trajectories calculated by the Fast Marching Method, it is possible to give two solutions:

First possibility, in order to avoid unrealistic trajectories, produced when the areas are narrower than the robot, the segments with distances to the obstacles and walls less than half the size of the robot need to be removed from the Voronoi Diagram previous to the Distance Transform.
Second possibility, used in this work, it is to dilate the objects and walls in a security distance that assure that the robot doesn't collide and doesn't accept passages narrower than the robot size.

The last step is to calculate the trajectory in the image generated by the Voronoi Diagram using the Fast Marching Method. Then, the path obtained verify the smooth and safety considerations required for mobile robot path planning.

The advantages of this method are the easy implementation, the speed of the method and the quality of the trajectories. The method works in $2 \mathrm{D}$ and $3 \mathrm{D}$, and it can be used at a global or local scale, in this case operating with sensor information instead of using an a priori map (sensor based planning).

\section{INTRODUCTION TO THE VORONOI DIAGRAM AND SKELETON}

It has been observed that the skeleton is embedded in the Voronoi diagram of a polygonal shape [2]. Similarly, the skeleton of a shape described by a discrete sequence of boundary points can be approximated from the Voronoi diagram of the points [3]. Both approaches yield a connected, Euclidean skeleton, but the latter is perhaps more appropriate for images since point sequences are more easily obtained than polygons. Although it is not true in general, if one restricts the shapes to those which are morphologically open and closed with respect to a finite-sized disk, the resulting skeleton approximated from the Voronoi diagram of a finite sampling of the boundary is close to the actual skeleton. In this case, the approximation error can be quantified, and be made arbitrarily close to zero.

\section{A. Voronoi Diagram and Skeleton.}

Consider the set $F$, closed in $R^{2}$. Associated with each point in $F$ is its Voronoi region.

$$
V_{F}(p)=\{q: d(q, p) \leq d(q, F /\{p\})\}
$$

The Voronoi diagram of $F$ is the union of the boundaries of all the Voronoi regions.

$$
V D(F)=\bigcup_{p \in F} \partial V_{F}(p) .
$$


A maximal disk in $G$ is one which is contained in $G$ while not being contained by any other disk in $G$. Assume that all maximal disks in $G$ are bounded. The skeleton $\sigma(G)$ is the set of centers of maximal disks in $G$. One desires the skeleton to be a "graph-like" retraction of the original set. In general, this cannot be assured due to the presence of infinitesimal detail. However, it is possible to eliminate these fine structures by assuming a reasonable subclass: the regular sets.

A compact set, $K$, is said to be $r$-regular [4] if it is morphologically open and closed with respect to a disk of radius $r>0$. It is possible to show that $\partial K$ is a disjoint union of closed simple $C^{2}$ curves with curvature magnitude no greater than $1 / r$. The skeleton of the interior of $K$ is well-behaved and graph-like.

\section{B. Skeleton-based generalization algorithm}

One issue that needs improvement is the existence of spurious hairs on the skeletons generated. This is a well-known artifact of skeleton generation, where any irregularities in the boundary generate unwanted skeleton branches. Ogniewicz [?] attempted to reduce skeletons formed from raster boundary points to a simple form by pruning the leaf nodes of the skeleton until a specified minimum circumcircle was achieved, but with the development of the one-step crust and skeleton algorithm this process may be greatly simplified. Alt and Schwartzkopf [5], as well as Blum [6] showed that leaf nodes of a skeleton correspond to locations of minimum curvature on the boundary. For a sampled boundary curve this means that three adjacent sample points are cocircular, with their centre at the skeleton leaf. If we wish to simplify the skeleton we should retract leaf nodes to their parent node location. This means that we now have four cocircular points instead of three. The retraction is performed by taking the central point of the three defining the leaf node, and moving it towards the parent node of the skeleton until it meets the parent node circumcircle. This smooths outward-pointing salients in the boundary of the object. The same should be done from the other side of the boundary, retracting those salients also. This may displace some of the points involved in the first smoothing step, but as the process is convergent a small number of iterations suffices to produce a smoothed curve having the same number of points as the original, but with a simplified skeleton.

\section{IntRoduction TO THE LEVEL SET METHOD AND THE FAST MARCHING METHOD}

The level set method was devised by Osher and Sethian as a simple and versatile method for computing and analyzing the motion of the interface in two or three dimensions. The goal is to compute and analyze the subsequent motion of the interface under a velocity field. This velocity can depend on position, time, the geometry of the interface and the external physics. The interface is captured for later time as the zero level set of a smooth (at least Lipschitz continuous) function. Topological merging and breaking are well defined and easily performed.
The original level set idea of Osher and Sethian (Osher [7]) for tracking the evolution of an initial front $\gamma_{0}$ as it propagates in a direction normal to itself with a given speed function $\mathrm{V}$. The main idea is to match the one-parameter family of fronts $\left\{\gamma_{t}\right\}_{t>0}$, where $\gamma_{t}$, is the position of the front at time $t$, with a one-parameter family of moving surfaces in such a way that the zero level set of the surface always yields the moving front. To determine the front propagation, we then need to find and solve a partial differential equation for the motion of the evolving surface. To be more precise, let $\gamma_{0}$ be an initial front in $\mathrm{R}^{d}, d \geq 2$ and assume that the so-called level set function $\phi: \mathrm{R}^{d} \times \mathrm{R}_{+} \rightarrow \mathrm{R}$ is such that at time $t \geq 0$ the zero level set of $\phi$ is the front $\gamma_{t}$. We further assume that $\phi(\boldsymbol{x} ; 0)= \pm d(\boldsymbol{x})$; where $d(\boldsymbol{x})$ is the distance from $\boldsymbol{x}$ to the curve $\gamma_{0}$. We use plus sign if $\boldsymbol{x}$ is inside 0 and minus if $\boldsymbol{x}$ is outside. Let each level set of $\phi$ along its gradient field with speed V. This speed function should match the desired speed function for the zero level set of $\phi$. Now consider the motion of, e.g., the level set

$$
\left\{\boldsymbol{x} \in \mathrm{R}^{d}: \phi(\boldsymbol{x} ; t)=0\right\} .
$$

Let $\boldsymbol{x}(t)$ be trajectory of a particle located at this level set so that

$$
\phi(\boldsymbol{x}(t) ; t)=0 .
$$

The particle speed $d \boldsymbol{x} / d t$ in the direction $\mathrm{n}$ normal to the level set is given by the speed function $V$, and hence

$$
\frac{d \boldsymbol{x}}{d t} \cdot n=V .
$$

where the normal vector $\mathrm{n}$ is given by

$$
n=-\frac{\nabla \phi}{|\nabla \phi|} .
$$

This is a vector pointing outwards, giving our initialization of $\mathrm{u}$. By the chain rule

$$
\frac{\partial \phi}{\partial t}+\frac{d \boldsymbol{x}}{d t} \cdot \nabla \phi=0
$$

Therefore $\phi(x ; t)$ satisfies the partial differential equation (the level set equation)

$$
\frac{\partial \phi}{\partial t}-V|\nabla \phi|=0
$$

and the initial condition

$$
\phi(\boldsymbol{x} ; t=0)= \pm d(\boldsymbol{x}) .
$$

This is called an Eulerian formulation of the front propagation problem because it is written in terms of a fixed coordinate system in the physical domain.

If the speed function $\mathrm{V}$ is either always positive or always negative, we can introduce a new variable (the arrival time function) $T(\boldsymbol{x})$ defined by

$$
\phi(\boldsymbol{x}, T(\boldsymbol{x}))=0
$$


In other words, $T(\boldsymbol{x})$ is the time when $\phi(\boldsymbol{x} ; t)=0$. If $\frac{d \boldsymbol{x}}{d t} \neq 0$, then $\mathrm{T}$ will satisfy the stationary Eikonal equation

$$
V|\nabla T|=1
$$

coupled with the boundary condition

$$
\left.T\right|_{d(\boldsymbol{x})=0}=0 \text {. }
$$

The advantage of this formulation 11 is that we can solve it numerically by the fast marching method [8], which is precisely what we will do in this paper.



Fig. 1. Map of the room used in the first experiment.

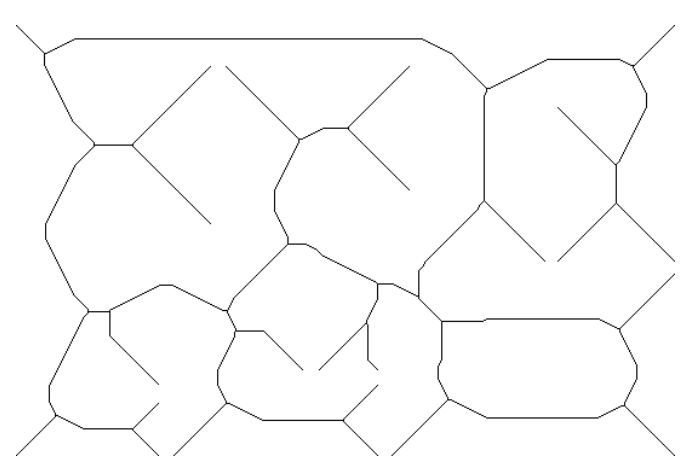

Fig. 2. Voronoi Diagram of the room.

Summing up, the central mathematical idea is to view the moving front $\gamma_{t}$ as the zero level set of the higher-dimensional level set function $\phi(x ; t)$. Depending on the form of the speed function $V$, the propagation of the level set function $\phi(x ; t)$ is described by the initial problem for a nonlinear HamiltonJacobi type partial differential equation 7 of first or second order.

If $V>0$ or $V<0$, it is also possible formulate the problem in terms of a time function $T(x)$ which solves a boundary value problem for a stationary Eikonal equation 11.



Fig. 3. Enlarged Voronoi Diagram of the room.

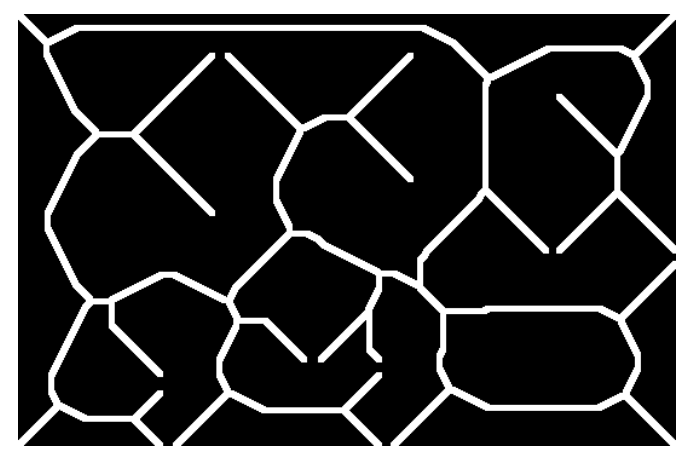

Fig. 4. Inverted image of the Enlarged Voronoi Diagram of the room.



Fig. 5. Trajectory calculated by Fast Marching Method in the inverted image of the Enlarged Voronoi Diagram of the room. 
Fast Marching Methods are designed for problems in which the speed function never changes sign, so that the front is always moving forward or backward. This allows us to convert the problem to a stationary formulation, because the front crosses each grid point only once. This conversion to a stationary formulation, plus a whole bunch of numerical tricks, gives it its tremendous speed

Level Set Methods are designed for problems in which the speed function can be positive in some places are negative in others, so that the front can move forwards in some places and backwards in others. While significantly slower than Fast Marching Methods, embedding the problem in one higher dimension gives the method tremendous generality.

Because of the nonlinear nature of the governing partial differential equation 7 or 11 , solutions are not smooth enough to satisfy this equation in the classical sense (the level set function and the time function are typically only Lipschitz). Furthermore, generalized solutions, i.e., Lipschitz continuous functions satisfying the equations almost everywhere, are not uniquely determined by their data and additional selection criteria (entropy conditions) are needed to pick out the (physically) correct generalized solutions. The correct mathematical framework in which to treat Hamilton-Jacobi type equations is provided by the notion of viscosity solutions (Crandall [9], [10]).

After its introduction, the level set approach has been successfully applied to a wide collection of problems that arise in geometry, mechanics, computer vision, and manufacturing processes, see ( Sethian [11]) for details. Numerous advances have been made to the original technique, including the adaptive narrow band methodology ( Adalsteinsson and Sethian [12]) and the fast marching method for solving the static Eikonal equation ( Sethian [13], [11]). For further details and summaries of level set and fast marching techniques for numerical purposes, see ( Sethian [11]). The Fast Marching Method is an $O(n \log (n))$ algorithm.

\section{IMPLEMENTATION OF THE METHOD}

This method operates in two steps. The first step starts with the calculation of the Voronoi Diagram of the $2 \mathrm{D}$ or $3 \mathrm{D}$ a priori map of the environment (which are the cells located equidistant to the obstacles). This process is done by means of morphological operations on the image of the environment map. To be more precise, it is done a skeletonization with the image techniques previously mentioned, in order to obtain the Voronoi Diagram. After that, a dilatation is done in order to have a thick Voronoi Diagram where to calculate the propagation of a wave front. This is done in order to obtain two characteristics, on one side the Voronoi Diagram has abrupt changes of gradient, especially in nodes and in other side Fast Marching Method used in next step, is a method designed for more than one dimension.

This way, the enlarged Voronoi Diagram will let the Fast Marching Method in the second step to plan the shortest trajectory. This trajectory is obtained inside the most safe areas provided by the enlarged Voronoi Diagram and properly smooth because the Fast Marching Method selects a continuous path in gradient terms.Besides the path extraction is very fast because the Fast Marching Method propagates in an almost unidimensional curve (it is not completely unidimensional due to the Voronoi Diagram is enlarged in the perpendicular direction to the Diagram curves in some cells).

After that,it is necessary to invert the image because it is necessary to have a viscosity map where the wave goes faster in the clearer zones and slower in the darker ones. The calculation of the evolution of the wave front is done with the Fast Marching Method.



Fig. 6. Trajectory calculated by Fast Marching Method directly, without the previous Voronoi Diagram. The trajectory is not safe because touches the corners and walls.

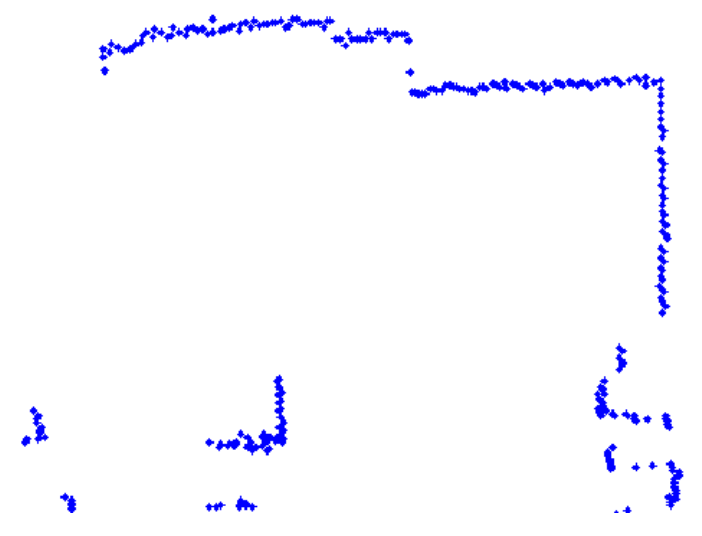

Fig. 7. Raw Laser data read by the robot (Local map).

The method proposed, can also be used for sensor based planning, working directly on a raw sensor image of the environment, as shown in figures 7 and 8 . 


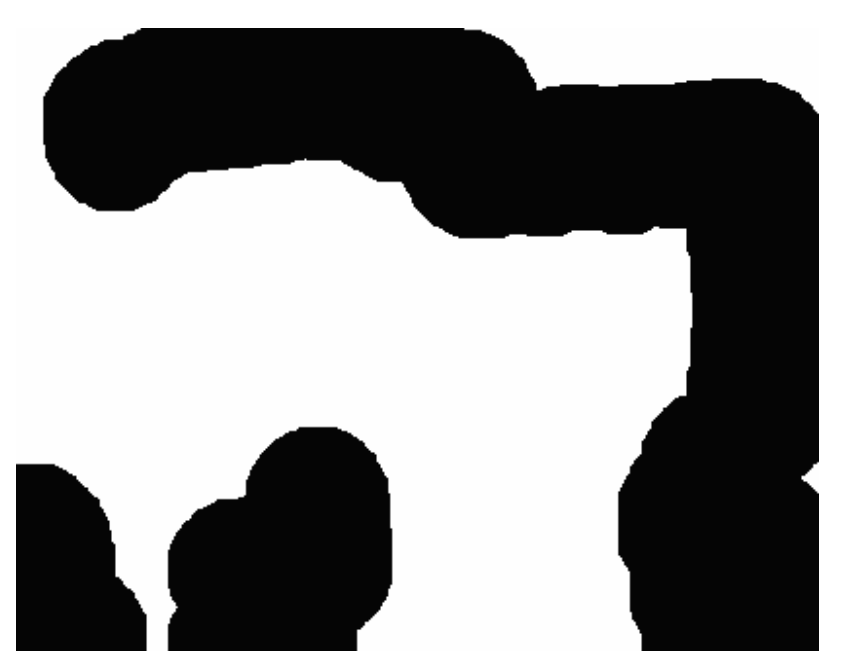

Fig. 8. Enlarged Laser data read by the robot (Local map).

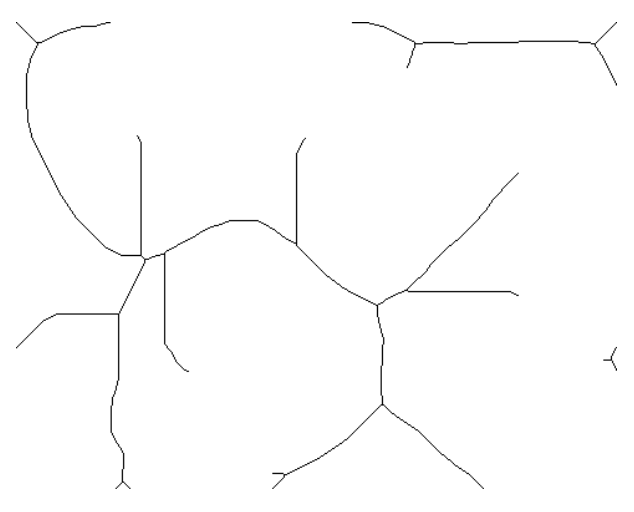

Fig. 9. Voronoi Diagram of the Enlarged Laser data read by the robot (Local map).

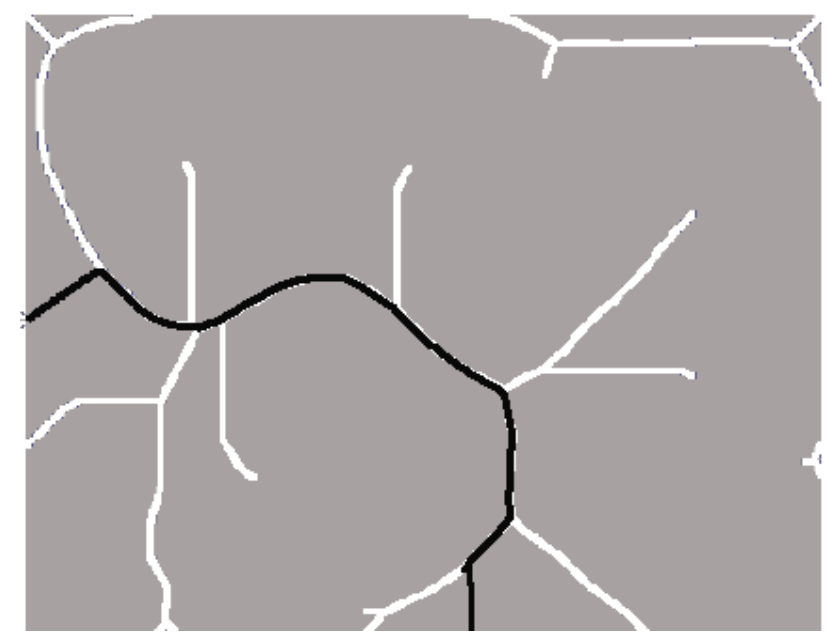

Fig. 10. Trajectory calculated with Fast Marching over the Voronoi Diagram of the Enlarged Laser data read by the robot (Local map).

\section{Results}

To illustrate the method possibilities, it has been used for planning a trajectory in difficult tests room (figures 1, 2, 3, 4 and 5), in local environments (figures 7, 8, 9 and 10) and in a typical offices's indoor environment as shown in figures 11 and 12. The dimensions of the environment are 116x14 meters (the cell resolution is $12 \mathrm{~cm}$ ). For this environment the first step (the Voronoi extraction) takes 0.06 seconds in a Pentium 4 at $2.2 \mathrm{Ghz}$, and the second step (Fast Marching) takes 0.20 seconds for a long trajectory.

The proposed method is highly efficient from a computational point of view because of the method operates directly over a 2D image map (without extracting adjacency maps), and due to the fact that Fast Marching complexity is $O(n \log (n))$ and the Distance Transform is also of complexity $O(n \log (n))$, where $n$ is the number of cells in the environment map.

The method provides smooth trajectories that can be used at low control levels without any additional smooth interpolation process.

The results are shown in fig 10 (The environment map of the Robotics Lab.) 11 and fig 12 (the path obtained after applying the Fast Marching method to the previous Voronoi diagram image).

\section{CONCLUSION}

The results obtained shows that the Voronoi Diagram can be used to improve the results obtained with Fast Marching method applied to Path Planning, to obtain smooth and safe trajectories.

The algorithm complexity is $O(n \log (n))$, where $n$ is the number of cells in the environment map, which let us use the algorithm on line. Besides, the algorithm can be used directly with raw sensor data to implement a sensor based local path planning.

\section{REFERENCES}

[1] J.-C. Latombe, Robot motion planning. Dordrecht, Netherlands: Kluwer Academic Publishers, 1991.

[2] D. T. Lee, "Medial axis transformation of a planar shape," IEEE Trans. on Pattern Analysis and Machine Intelligence, vol. 4, no. 4, pp. 398-401, 1982.

[3] P. K. J. D. Boissonnat, "Use of the delaunay triangulation for the identification and localization of objects," IEEE Conf. Computer Vision and Pattern Recognition, pp. 398-401, 1985.

[4] J. Serra, Image Analysis and Mathematical Morphology. London: Academic Press, 1982.

[5] O. S. H. Alt, "The voronoi diagram of curved objects," in Proc. 11th Annual ACM Symposium on Computational Geometry, 1995, pp. 89-97.

[6] H. Blum, "A transformation for extracting new descriptors of shape," in Models for Preception of Speech and Visual Form, W. W. Dunn, Ed. MIT Press, Cambridge, Mass., 1967, pp. 153-171.

[7] S. Osher and J. A. Sethian, "Fronts propagating with curvaturedependent speed: Algorithms based on hamilton-jacobi formulations," Journal of Computational Physics, no. 79, pp. 12-49, 1988.

[8] J. A. Sethian, "A fast marching level set method for monotonically advancing fronts," Proc. Nat. Acad Sci. U.S.A., vol. 93, no. 4, pp. 1591$1595,1996$.

[9] P. L. L. M. G. Crandall, H. Ishii, "User's guide to viscosity solutions of second order partial differential equations," Bull. Amer. Math. Soc., vol. 27, no. 1, pp. 1-67, 1992.

[10] P. L. L. M. G. Crandall, "Viscosity solutions of hamilton jacobi equations," Trans. Amer. Math. Soc., vol. 277, pp. 1-42, 1983. 


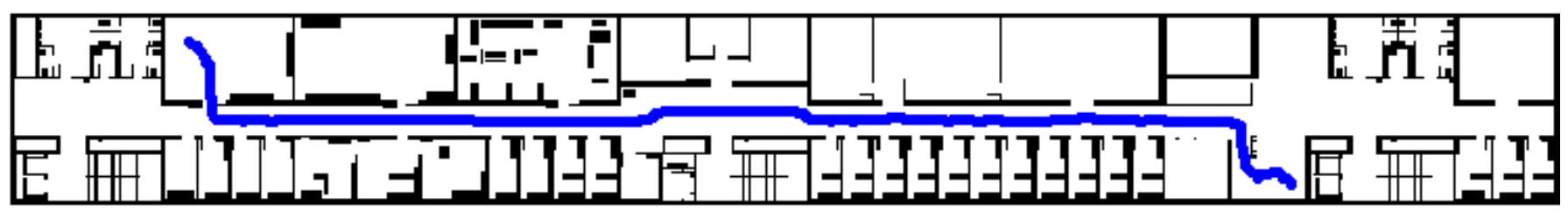

Fig. 11. Trajectory calculated with the presented method and the original map (Global map).

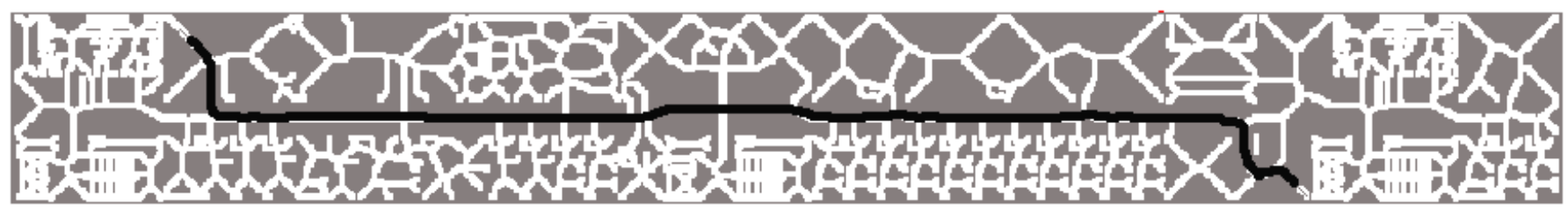

Fig. 12. Trajectory calculated with Fast Marching over the Voronoi Diagram (Global map).

[11] J. A. Sethian, "Theory, algorithms, and aplications of level set methods for propagating interfaces," Acta numerica, pp. 309-395, 1996, cambridge Univ. Press.

[12] D. Adalsteinsson and J. Sethian, "A fast level set method for propagating interfaces," J. Comput. Phys., vol. 118, no. 2, pp. 269-277, 1995.

[13] J. Sethian, Level set methods. Cambridge University Press, 1996. 